\title{
Retiform Hemangioendothelioma of the Neck
}

\author{
Chin-Lung Kuo ${ }^{1,2,3,4} \cdot$ Paul Chih-Hsueh Chen ${ }^{5,6} \cdot$ Wing-Yin Li ${ }^{5,6} \cdot$ Pen-Yuan Chu ${ }^{1,3}$ \\ 1Department of Otorhinolaryngology-Head and Neck Surgery, Taipei Veterans General Hospital, Taipei; ${ }^{2}$ Department of Otorhinolaryngology, Taoyuan Armed Forces General \\ Hospital, Taoyuan; ${ }^{3}$ Department of Otorhinolaryngology, ${ }^{4}$ nstitute of Brain Science, National Yang-Ming University, Taipei; ${ }^{5}$ Department of Pathology and Laboratory \\ Medicine, Taipei Veterans General Hospital, Taipei; ${ }^{\circ}$ Department of Pathology, National Yang-Ming University School of Medicine, Taipei, Taiwan
}

Vascular tumors are categorized into benign hemangiomas, frankly malignant angiosarcomas, and tumors of intermediate malignancy. Hemangioendotheliomas are of intermediate malignancy and encompass a broad range of histologic entities, including retiform, epithelioid, kaposiform, polymorphous, and composite sub-types. ${ }^{1}$ Retiform hemangioendothelioma (RH) is an extremely rare disease entity of unknown etiology that was first described by Calonje et al..$^{2}$ in 1994 as a vascular neoplasm of intermediate malignancy. It is characterized by a high rate of local recurrence and a low frequency of metastasis, and its biologic potential is between that of hemangiomas and angiosarcomas. RH reportedly presents primarily as an exophytic dermal tumor of the extremities. ${ }^{3}$ We present the first report of $\mathrm{RH}$ arising in the neck without dermal involvement.

\section{CASE REPORT}

A previously healthy 27 -year-old woman presented to the out-patient department of our institution in February 2012 with a painless mass on the left side of the neck, which had increased in size over the course of one year. She had no constitutional symptoms such as weight loss, fever, or decreased appetite, and the rest of her medical history was also unremarkable. Physical examination revealed a $4 \times 3-\mathrm{cm}$ mass occupying the left side of the neck at the level III lymph node region. On palpation, the lesion was non-tender, hard, movable, and showed well-defined and smooth borders. The overlying skin was nor-

\section{Corresponding Author}

Pen-Yuan Chu, M.D.

Department of Otorhinolaryngology-Head and Neck Surgery, Taipei Veterans General Hospital, 201, Sec 2, Shih-Pai Road, Taipei 112, Taiwan

Tel: +886-2-2875-7337, Fax: +886-2-2875-7338, E-mail: pychu@vghtpe.gov.tw

Received: June 11, 2013 Revised: October 6, 2013

Accepted: October 14, 2013 mal. Cervical lymph node enlargement was not observed, and the remainder of the otolaryngopharyngeal examination was unremarkable. Ultrasound-guided fine-needle aspiration was performed, and the cytology results were negative for malignancy. Computed tomography of the neck showed a well-defined heterogeneous mass with prominent ring enhancement occupying the left side of the neck at the level III lymph node region (Fig. 1). Surgical excision revealed a well-encapsulated and hypervascular tumor within the left anterior neck triangle.

Histologic examination revealed a $3.8 \times 2 \times 2$-cm solid tumor with clear surgical margins. The tumor was composed of vascular structures containing elongated and narrow arborizing vascular channels simulating the structure of a rete testis (Fig. 2). The vascular channels were lined with hobnail endothelial cells (Fig. 3), which demonstrated rare mitosis. The tumor cells were immune-reactive for $\mathrm{CD} 31$ and friend leukemia virus integration 1 (Fli-1), but not for smooth muscle actin. Based on these histopathologic and immunohistochemical features, a diagnosis of $\mathrm{RH}$ was reached.

After discussion with the head and neck oncology multidisciplinary team, a regular follow-up program was scheduled for the patient without postoperative radiation or chemotherapy, because of the clear surgical margins and rare mitosis observed, and to avoid the potential life-long complications associated with radiation therapy. No recurrence was evident at 1-year follow-up.

\section{DISCUSSION}

To date, only 32 cases of $\mathrm{RH}$ have been reported in the English literature. ${ }^{4}$ The etiology of this disease remains unclear; although cases related to previous epidermal malignant neoplasms, radiotherapy, human herpes virus type 8 and lymphedema have 

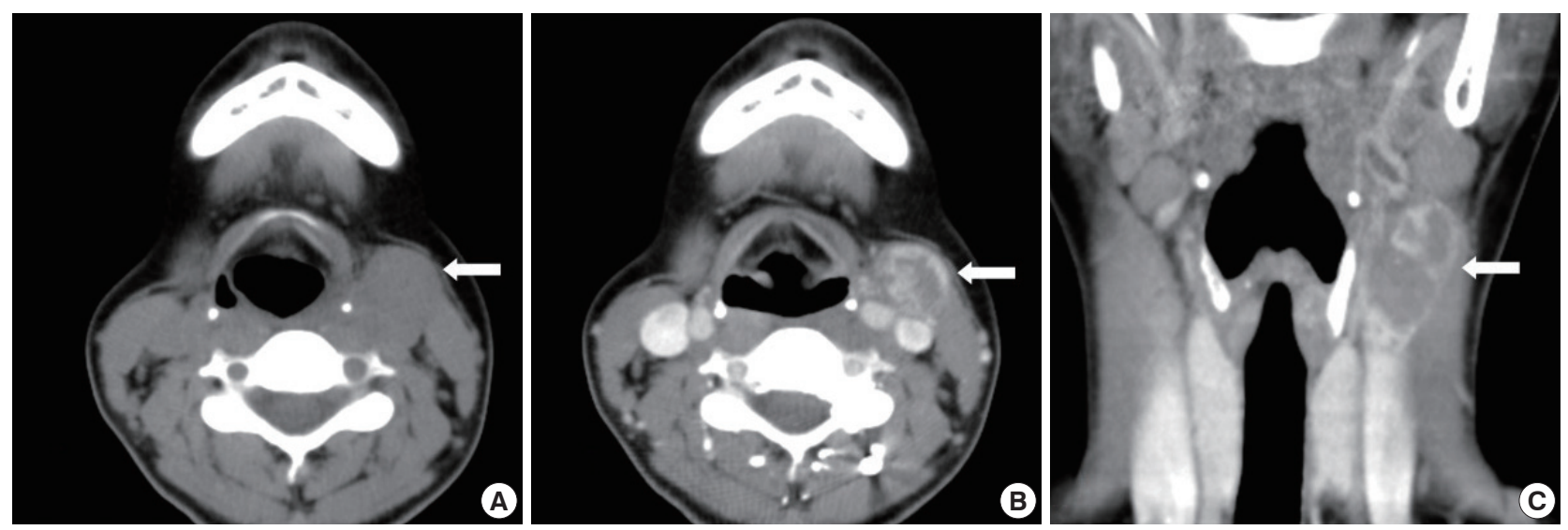

Fig. 1. Computed tomography without contrast shows a mass occupying the left side of the neck at the level III lymph node region (A, arrow); with contrast, the well-defined mass reveals heterogeneous enhancement, with prominent ring enhancement (arrows) (B, C).

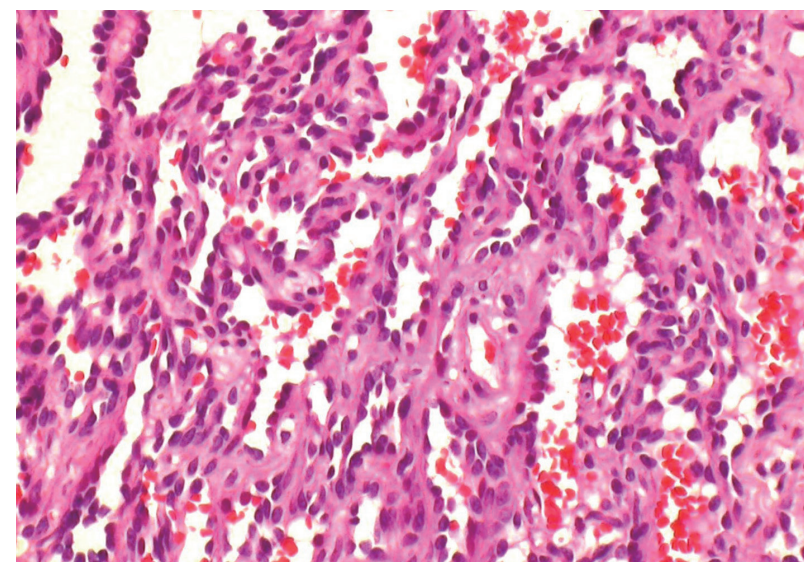

Fig. 2. Tumor histopathology shows complex branching vessels, resembling a rete testis.

been reported, the exact associations have not been clearly established. ${ }^{2,45}$ Disease duration from the time of diagnosis ranges from 2 months to several years. ${ }^{2,3,6}$ Most patients present between the second to fourth decades of life (mean age, 36 years). ${ }^{2}$ There is a female predominance, with a female-to-male ratio of $2: 1 ., 3,6 \mathrm{RH}$ can develop as either exophytic or plaque-like lesions with size ranging from 1 to $30 \mathrm{~cm} .{ }^{3,6}$ Most cases appear in the extremities, with a higher incidence in the lower limbs. Occasionally, this disease occurs in the trunk, scalp, pinna, and penis. ${ }^{2-5} \mathrm{An} \mathrm{RH}$ presenting as a neck mass, as seen in this case, is unusual and has not been previously reported. The differential diagnoses generally include angiosarcoma, papillary intralymphatic angioendothelioma (PILA), hobnail hemangioma and Dabska-RH. ${ }^{3-5,7,8}$

The most important differential diagnosis of $\mathrm{RH}$ is angiosarcoma because of the therapeutic and prognostic considerations. Angiosarcoma is an aggressive neoplasm with a high mortality

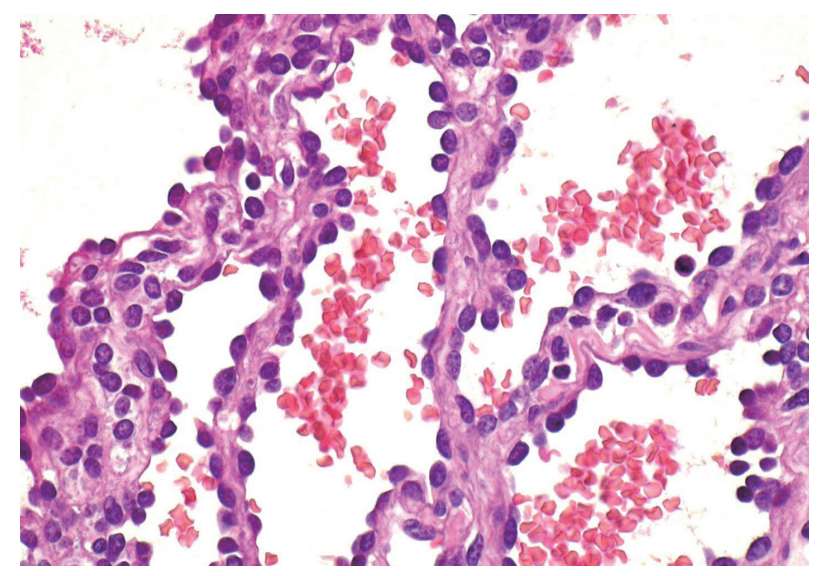

Fig. 3. The vessels are lined by monomorphic hobnail-like endothelial cells, without significant morphologic atypia.

rate and a very high incidence of local recurrence and metastasis. $^{6} \mathrm{RH}$ also exhibits frequent local recurrence, but rarely metastasizes, ${ }^{2,3}$ and no tumor-related deaths have been reported. ${ }^{6}$ The vessels of angiosarcoma usually do not have a retiform appearance. Angiosarcoma is characterized by more nuclear atypia, conspicuous mitotic activity, tumor infiltration between individual collagen bundles, and multi-layering of endothelial cells without hobnail morphology. ${ }^{3,6}$ PILA is another important differential diagnosis of $\mathrm{RH}$. Although the histologic features of RH overlap with those of PILA, PILA lacks a retiform growth pattern, and is characterized by cavernous lymphangioma-like vascular spaces. Unlike $\mathrm{RH}$, which typically occurs in the extremities, PILA shows no preference for a specific anatomic site. ${ }^{7}$ Hobnail hemangioma can be confused with RH due to its similar hobnail endothelial cells. Hobnail hemangioma, however, lacks the complex retiform vessels and is a circumscribed tumor. Furthermore, the hobnail endothelial cells of hobnail hemangi- 
oma are seen in only the most superficial vessels. Collagen dissection is usually present, and an inflammatory infiltrate is not usually prominent. ${ }^{3}$ Dabska-retiform hemangioendothelioma and $\mathrm{RH}$ are closely related tumors characterized by the presence of "hobnail"-type endothelial cells. Although RH typically appears in young to middle-age adults in the lower limbs and trunk, Dabska tumors have no particular pattern in terms of age or anatomical site. In addition, $\mathrm{RH}$ is considered to be a vascular tumor, while Dabska-RHs have a lymphatic endothelial phenotype. $^{8}$

Cytologic or pathological examination reports may provide surgeons with appropriate guidelines for the treatment of patients, and the most compelling evidence in the differential diagnosis of $\mathrm{RH}$ is provided by immunohistochemistry, in which tumor cells react with endothelial markers (e.g., CD31, Fli-1, and factor VIII-related antigen). ${ }^{2,5,9,10} \mathrm{CD} 31$ is generally regarded as the single best marker of endothelial cell differentiation because it is expressed in $90 \%$ of endothelial cell tumors, but very rarely in carcinomas, lymphomas, and mesotheliomas. ${ }^{9}$ Factor VIII-related antigen has low sensitivity, and frequent presence of significant "background" due to staining of circulating antigen greatly limits the use of this marker. ${ }^{10}$ Hence, the expression of this marker was not determined in this patient.

There are differences between the present case and those in previous reports of $\mathrm{RH}$ in terms of disease location (neck vs mainly extremities), morphology (encapsulated mass vs mainly exophytic lesion), and histology (deep soft tissue vs mainly dermal involvement). These differences may expand the understanding of RH. In summary, there can be diagnostic pitfalls in general practice when managing neck masses, particularly an uncommon tumor in an unusual site. Awareness of this unique RH with uncertain malignant behavior is essential. In addition, as the prognosis and adjuvant therapy for $\mathrm{RH}$ and angiosarcoma are very different, it is important to distinguish them histopathologically.

\section{Conflicts of Interest}

No potential conflict of interest relevant to this article was reported.

\section{Acknowledgments}

We thank the Department of Pathology and Laboratory Medicine, Taipei Veterans General Hospital, Taipei, Taiwan, R.O.C. for assistance in the manuscript preparation.

\section{REFERENCES}

1. Chu YC, Choi SJ, Park IS, Kim L, Han JY, Kim JM. Composite hemangioendothelioma: a case report. Korean J Pathol 2006; 40: 142-7.

2. Calonje E, Fletcher CD, Wilson-Jones E, Rosai J. Retiform hemangioendothelioma: a distinctive form of low-grade angiosarcoma delineated in a series of 15 cases. Am J Surg Pathol 1994; 18: 115-25.

3. Tan D, Kraybill W, Cheney RT, Khoury T. Retiform hemangioendothelioma: a case report and review of the literature. J Cutan Pathol 2005; 32: 634-7.

4. O'Duffy F, Timon C, Toner M. A rare angiosarcoma: retiform haemangioendothelioma. J Laryngol Otol 2012; 126: 200-2.

5. Ioannidou D, Panayiotides J, Krasagakis K, Stefanidou M, Manios A, Tosca A. Retiform hemangioendothelioma presenting as bruise-like plaque in an adult woman. Int J Dermatol 2006; 45: 53-5.

6. Hirsh AZ, Yan W, Wei L, Wernicke AG, Parashar B. Unresectable retiform hemangioendothelioma treated with external beam radiation therapy and chemotherapy: a case report and review of the literature. Sarcoma 2010; 2010: 756246.

7. Fanburg-Smith JC, Michal M, Partanen TA, Alitalo K, Miettinen M. Papillary intralymphatic angioendothelioma (PILA): a report of twelve cases of a distinctive vascular tumor with phenotypic features of lymphatic vessels. Am J Surg Pathol 1999; 23: 1004-10.

8. Yarmel D, Dormans JP, Pawel BR, Chang B. Recurrent pedal hobnail (Dabska-retiform) hemangioendothelioma with forefoot reconstructive surgery using a digital fillet flap. J Foot Ankle Surg 2008; 47: 487-93.

9. Pusztaszeri MP, Seelentag W, Bosman FT. Immunohistochemical expression of endothelial markers CD31, CD34, von Willebrand factor, and Fli-1 in normal human tissues. J Histochem Cytochem 2006; 54: 385-95.

10. Billings SD, Folpe AL, Weiss SW. Epithelioid sarcoma-like hemangioendothelioma. Am J Surg Pathol 2003; 27: 48-57. 